\title{
Diagnosing Ventilator-Associated Pneumonia (VAP) In UK NHS ICUs: The Perceived Value And Role of A Novel Optical Technology
}

William Stephen Jones ( $\nabla$ will.jones@ncl.ac.uk)

Newcastle upon Tyne Hospitals Foundation Trust https://orcid.org/0000-0001-9352-3916

Jana Suklan

Newcastle University

Amanda Winter

Newcastle upon Tyne Hospitals Foundation Trust

Kile Green

Newcastle upon Tyne Hospitals Foundation Trust

Tom Craven

University of Edinburgh

Annya Bruce

University of Edinburgh

Joanne Mair

University of Edinburgh

Kev Dhaliwal

University of Edinburgh

Tim Walsh

University of Edinburgh

John Simpson

Newcastle upon Tyne Hospitals Foundation Trust

Sara Graziadio

Newcastle upon Tyne Hospitals Foundation Trust

Joy Allen

Newcastle University

\section{Research Article}

Keywords: ventilator-associated pneumonia, VAP, interviews, care pathway analysis, thematic analysis

Posted Date: October 25th, 2021

DOI: https://doi.org/10.21203/rs.3.rs-956278/v1 
License: (c) (i) This work is licensed under a Creative Commons Attribution 4.0 International License. Read Full License

Version of Record: A version of this preprint was published at Diagnostic and Prognostic Research on February 10th, 2022. See the published version at https://doi.org/10.1186/s41512-022-00117-x. 


\section{Diagnosing ventilator-associated pneumonia (VAP) in UK 2 NHS ICUs: the perceived value and role of a novel optical 3 technology}

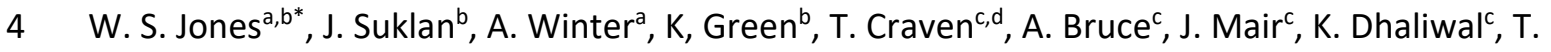

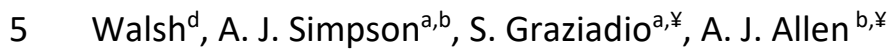

6 a NIHR Newcastle In Vitro Diagnostics Co-operative, Newcastle upon Tyne Hospitals Foundation Trust, 7 Newcastle upon Tyne, NE1 4LP, United Kingdom

b NIHR Newcastle In Vitro Diagnostics Co-operative, Translational \& Clinical Research Institute, Newcastle University, Newcastle upon Tyne, NE2 4HH, United Kingdom

c Translational Healthcare Technologies Group, Queen's Medical Research Institute, University of Edinburgh, Edinburgh EH16 4TJ, United Kingdom

${ }^{\mathrm{d}}$ Edinburgh Critical Care Research Group, University of Edinburgh, Edinburgh, United Kingdom

${ }^{*}$ Corresponding author, email: will.jones@ncl.ac.uk

$¥$ Joint last authors, equal contribution 


\section{Background}

24

25

Diagnosing ventilator-associated pneumonia (VAP) in an intensive care unit (ICU) is a complex process. Our aim was to collect, evaluate and represent the information relating to current clinical practice for the diagnosis of VAP in UK NHS ICUs, and to explore the potential value and role of a novel diagnostic for VAP, which uses optical molecular alveoscopy to visualise the alveolar space.

\section{Methods}

Qualitative study performing semi-structured interviews with clinical experts. Interviews were recorded, transcribed, and thematically analysed. A flow diagram of the VAP patient pathway was elicited and validated with the expert interviewees. 14 clinicians were interviewed from a range of UK NHS hospitals: 12 ICU consultants, 1 professor of respiratory medicine and 1 professor of critical care.

\section{Results}

Five themes were identified, relating to: [1] current practice for the diagnosis of VAP; [2] current clinical need in VAP diagnostics; [3] the potential value and role of the technology; [4] the barriers to adoption; and [5] the evidence requirements for the technology, to help facilitate successful adoption. These themes indicated that diagnosis of VAP is extremely difficult, as is the decision to stop antibiotic treatment. The analysis revealed that there is a clinical need for a diagnostic that provides an accurate and timely diagnosis of the causative pathogen, without the long delays associated with return of culture results, and which is not dangerous to the patient. It was determined that the technology would satisfy important aspects of this clinical need for diagnosing VAP (and pneumonia, more generally), but would require further evidence on safety and efficacy in the patient population to facilitate adoption.

\section{Conclusions}

Care pathway analysis performed in this study was deemed accurate and representative of current practice for diagnosing VAP in a UK ICU as determined by relevant clinical experts, and explored the value and role of a novel diagnostic, which uses optical technology, and could streamline the diagnostic pathway for VAP and other pneumonias.

\section{Key words:}

ventilator-associated pneumonia, VAP, interviews, care pathway analysis, thematic analysis. 


\section{Introduction}

Pneumonia is a bacterial, viral, or fungal infection of the lungs, which causes the alveoli of the lungs to fill up with microorganisms, fluid, and inflammatory cells, preventing the lungs from functioning effectively [1]. The classification scheme for pneumonia in UK NHS hospitals is based on the setting in which the infection was mostly likely acquired: community-acquired pneumonia (CAP), when a patient is in the hospital $<48 \mathrm{hrs}$ before the pneumonia is suspected; hospital-acquired pneumonia (HAP), when a patient is in the hospital $>48 \mathrm{hrs}$ before the pneumonia develops, but is not associated with mechanical ventilation; and ventilator-associated pneumonia (VAP), when a patient is mechanicallyventilated and intubated for $>48 \mathrm{hrs}$ before the pneumonia develops [2-4]. The causative pathogens in each scenario are different, and treatment strategies vary as a consequence.

Acquiring a pneumonia in the ICU can have severe consequences for the patient. For example, VAP in the ICU is the leading cause of death relating to infection [3,5-7], and is associated with increased duration of mechanical ventilation, length of stay (in the ICU and hospital), morbidity and healthcare costs [8-11].

With the rise of novel coronavirus SARS-CoV-2 and the ensuing global pandemic, many countries have reported a sharp rise in ventilator use in ICU, prompting governments and health services to order vast quantities of ventilators to meet the growing demand. In the UK, initial estimates of ventilators required to deal with this influx stood at 30,000 in early March 2019 before being revised in April 2019 to 18,000 , still 10,000 units more than were in use in UK NHS practices at the start of that year [12]. A report from the Intensive Care National Audit described two-thirds of COVID-19 patients in the UK requiring critical care were put on mechanical ventilation within 24 hours of admission with a median length of stay of 3-5 days depending on the level of support required [13]. The increased use of ventilators, driven in part by the spread of COVID-19, will likely have an ongoing impact on the number of reported cases of VAP and bring about greater pressure for accurate and timely diagnosis of VAP.

The exponential growth in scientific and technological advancement has led to the development of several novel devices for the diagnosis of infections (in general) and pneumonia (in particular) [1422].The Translational Healthcare Technologies group [23], have developed an optical molecular alveoscopy (OMA) platform for the potential diagnosis of pneumonia at the bedside in the ICU setting (Figure 1). The OMA platform administers SmartProbes (microdoses of optical molecular imaging reagents that are delivered into the distal lung) to detect infection and inflammation, in real-time. These molecules fluoresce/light up when they bind to specific targets such as bacteria or activated neutrophils. Current clinically developed SmartProbes are specific for some gram-negative bacteria, 
gram-positive bacteria, and/or neutrophils. These Smartprobes are delivered through a multifunctional bundle (Panoptes fibre) that has been extended through serial transbronchial passes into the alveolar sacs, via the working channel of a bronchoscope. Panoptes is a triple lumen optical imaging, delivery and sampling device comprised of two delivery/aspiration capillaries and an imaging fibre. Two imaging systems (Versicolour and Kronoscan) support the real-time visualization of fluorescent bacteria and activated neutrophils within the patient's alveolar spaces. The OMA platform also has the capacity to perform a mini-lavage by extracting small volumes of liquid instilled in the alveolar space, which could be used for culturing and confirmation of infection.

Figure 1: Image showing a bronchoscopy procedure with the imaging fibre and capillary bundle being passed down the working channel of a bronchoscope into the alveolar regions. SmartProbes are delivered via the capillaries and images are sent via the imaging fibre to the imaging system at the patient's bedside.

The accuracy and utility of a diagnostic test is not a fixed property, but is dependent on the clinical setting and patient population in which it is used [24]. To establish the accuracy and clinical utility of a diagnostic device it is first necessary to understand the current diagnostic practice/pathway for the disease of interest.

Care pathway analysis, in the context of diagnostics, involves the collection, evaluation and representation of information relating to the diagnostic journey (the pathway) a patient group follows as part of a healthcare system [25-29]. In this study, we performed a set of qualitative, semi-structured interviews with clinical experts in order to conduct a care pathway analysis of the current practice for diagnosing VAP in UK NHS ICUs.

Understanding the pathway helps to determine the optimal role, setting and patient population for a new diagnostic, as well as the barriers and facilitators to adoption and future evidence requirements [27]. As part of this pathway analysis we also explored the potential value and role of the OMA platform in the diagnosis of VAP in the ICU. Broadly speaking, there are 4 possible roles for medical tests: (1) Screening, to determine if asymptomatic individuals have a disease, sometimes called Surveillance in ICUs; (2) Diagnostic, to determine if an individual has a disease; (3) Prognostic, to predict the likelihood of an individual developing a disease or deteriorating; and (4) Monitoring, to determine whether a patient's disease is controlled or is responding to treatment. Depending on how a test will be used it may also be further categorised as a rule-in or a rule-out test [27]. A rule-in test typically requires high specificity (low false positive rate), so that most non-diseased subjects will be diagnosed as non-diseased. Therefore, a positive result makes the presence of disease likely, effectively ruling-in disease. A rule-out test typically requires high sensitivity, so that most diseased subjects will be diagnosed as diseased. Therefore, a negative result essentially rules-out the disease 
in question. Rule-in tests are important when confirming a diagnosis following other clinical data or when subsequent tests or treatments are dangerous to the patient. Rule-out tests are important when there are severe consequences for missing a disease.

Recently, Korevaar et al [24] have recommended that in evaluating a medical test one consider where

122 it would be placed, and how it will affect the current pathway: whether it is a triage test, with the results determining which patients will undergo the existing test(s); an add on test, used before or after an existing test(s) to improve accuracy; a replacement test, replacing the existing test(s), expected to be more accurate, less invasive, less costly or more usable than the test replaced; and a new test, where a completely new test is added to the pathway, were there was not one previously. This consideration will be referred to as its 'role in the pathway', to distinguish it from its 'role as a medical test', described in the previous paragraph. In this study, we sought to elicit information on the optimal role (in both senses) of the OMA platform for use with suspected VAP patients in UK NHS ICUs.

\section{Methods}

\section{Interview structure}

Semi-structured interviews were conducted with 14 clinicians from a range of UK NHS hospitals: 12 ICU consultants, 1 professor of respiratory medicine and 1 professor of critical care. Interviews lasted between 45-60 minutes in length. Interviews took place in 2019. They were a mixture of face-to-face and telephone interviews and were performed by WSJ and JS. A topic guide was developed prior to the interviews (see Online Material, Appendix 1). Participants were invited to be interviewed via the UK Critical Care Research Group (UKCCRG) mailing list. The sample size used in this study ( $N=14$ ) was selected based on the concept of data saturation [30] and is consistent with previously published, qualitative research [25].

During the interviews, participants were first asked questions on the current care pathway for the diagnosis of VAP in their ICU. Next, participants watched a short video demonstrating the OMA platform (See Online Material); showing the same video to all participants reduced the potential for bias. Finally, after watching the video, the participants were asked questions about the potential value and role of the OMA platform in the diagnosis of a suspected VAP in the ICU. 
147 The research question and outcome measures in this study were not informed by patients' priorities,

148 experience, and/or preferences. Patients were not involved in study design. Patients were not

149 involved in recruitment to, or the conduct of, this study. Patient engagement is part of the

150 dissemination strategy. The research team plans to present the results of this study at various

151 meetings and conferences, where patients will be present.

152 Data analysis

153 Thematic analysis was used to identify the relevant themes from the interviews [31-34]. We used the 154 Gale et al. Framework Analysis approach [35]; see Online Material, Appendix 2 for details of this 155 approach.

157 The interview responses from the participants and the results from the thematic and care pathway analysis are synthesised below, divided into 5 key themes. Supporting quotes for each theme are available in the Online Material, Appendix 3.

\section{Theme 1: Current practice for the diagnosis of VAP}

161 At the beginning of the interviews, participants were asked to describe the current pathway for 162 diagnosing a suspected VAP in their ICU, including information on general ICU functioning.

164 ICUs, sometimes called critical care units or intensive therapy units, are specialist hospital wards that 165 care for severely ill patients who are closely monitored, often with a one to one, or one to two, 166 nurse/patient ratio.

167 Ward rounds occur at least twice daily, and involve a multidisciplinary team of physicians, nurses, 168 microbiologists and other allied healthcare providers. During the ward round the clinical team will 169 review the patient's clinical characteristics. This review will include the results of the non-specific, 170 routine investigations, which typically include blood, urine and sputum tests. 
172 Diagnosing a VAP in the ICU is challenging. The initial suspicion of VAP is based on clinical signs associated with the respiratory system, which are not specific to VAP. The typical clinical signs are: high/low temperature, leucocytosis/leukopenia, worsening oxygenation, gas exchange or increasing oxygen requirements, new infiltrates on chest x-ray (CXR) or computerised tomography (CT), suggestive auscultation, general worsening in haemodynamic state, increase in purulent sputum,

177 (colour, thickness and/or frequency), increase in c-reactive protein (CRP), drop in blood pressure, drop 178 in platelet count, and others. Besides pneumonia, these signs can also be indicative of 179 atelectasis/collapsed lung/pneumothorax, sepsis, major trauma (e.g. lung or brain injury), cardiogenic 180 pulmonary edema, pulmonary haemorrhage/embolism/fibrosis, cystic fibrosis, pleural effusion, acute 181 respiratory distress syndrome (ARDS), chronic obstructive pulmonary disease (COPD), mucous 182 impaction and other sources of infection. Several clinical scoring systems exist which aim to provide a 183 semi-objective clinical shortcut to the decision to initiate antibiotics for VAP, the Clinical Pulmonary 184 Infection Score (CPIS) being the most widely used and studied [36]. In general, these scores have 185 unsuitable test characteristics compared to microbiological confirmation and the use of CPIS to guide 186 antibiotic decisions is not recommended by the Infectious Diseases Society of America (IDSA) due to pooled sensitivity and specificity of $65 \%(95 \% \mathrm{Cl}$ : $61 \%$ to $69 \%)$ and $64 \%$ (95\% $\mathrm{Cl}$ : $60 \%$ to $67 \%$ ) respectively $[37,38]$. Retrospectively applied surveillance definitions are widely used because of the use of VAP as a quality indicator and are useful for benchmarking across populations. They have good face validity, making them tempting reference standards, but they may be gamed through interpretation of radiology [39] or timing of microbiology sampling [40], and exhibit low case concordance [41]. Also, the use of these scoring and recording systems is not always feasible in clinical practice, because it is logistically difficult to reliably record this information in the ICU.

To improve the accuracy of a diagnosis of suspected VAP requires the performance of an invasive diagnostic procedure, where an upper or lower respiratory specimen is collected and sent for microbiological testing. These procedures are sometimes referred to as special investigations. The correct special investigation to perform is still a matter of debate, with different ICUs employing different procedures [42]. The main procedures are: endotracheal aspirate (ETA), nonbronchoscopic bronchoalveolar lavage (N-BAL), protected specimen brush (PSB), and bronchoalveolar lavage (BAL). 
202 The treatment for a suspected VAP is antibiotics. The key driver for starting antibiotics is an overall, holistic deterioration in the clinical signs associated with the respiratory system. No single clinical sign or microbiological result is individually sufficient to initiate the decision to treat. Results from special investigations take time to return (up to 72 hours), because of inherent technical limitation in culturebased methods. Consequently, antibiotic treatment is typically started on the basis of clinical signs only. In this situation, treatment is with broad spectrum antibiotics. The choice of empirical antibiotic is protocolised in an ICU, in accordance with background resistance rates and influenced by individual patient characteristics. If recent respiratory microbiological isolates are available, they may be used to guide initial treatment, and if microbiology is acquired after initial treatment (i.e. from special investigations), it will be used to tailor a patient's antibiotics to the causative pathogen, if one is present. The advantages of tailoring antibiotics are several: it leads to more effective killing of bacteria, reduced exposure of patients to unnecessary toxicity (causing Clostridium difficile, etc.)[42], reduced risk of developing antimicrobial resistance (AMR) and reduced costs to the unit and healthcare system. There are no disadvantages to appropriately narrowed antibiotics, but if antibiotics are narrowed incorrectly the bacteria may survive the treatment, with dangerous consequences for the patient. Antibiotics are usually started with a set duration, typically a 5- or 7-day course. This course is strictly completed, unless the antibiotics are narrowed, an alternative diagnosis is confirmed, or if the patient is moving to a palliative mode of care.

The key driving factor for stopping antibiotics in a suspected VAP patient is an overall, holistic improvement in the patient, based on the clinical signs associated with the respiratory system. Deciding the exact point at which to stop antibiotics was reported to be extremely challenging, partly because there is no good rule-out or monitoring test for pneumonia, but also because there are no standardised and implemented local NHS guidelines to guide clinical decision making; see Online Material, Appendix 4 for a review of guidelines for VAP.

Cognitive bias also plays a role in this context. For example, it was reported in the interviews that in the ICU there is a tendency to start with broad-spectrum therapy and maintain this treatment regime, irrespective of whether the patient is getting better or not; If the patient is getting better, then it is assumed that the broad-spectrum antibiotic is working, so they stay broad, and if the patient is getting worse, they may also stay broad and even add on additional antibiotics. It was reported that during handovers between ICU consultants, it is very unlikely that the new consultant will stop antibiotics, 
even if the plan was to stop them on that day. They are likely to continue for a further $24 / 48$ hours to confirm in their own mind that the patient is clinically well enough to stop. It was also reported that antibiotics are often the only treatment available to help an ICU patient recover from illness, so they are check-mated into continuing antibiotics beyond the conventional course length. This is compounded by the ease and cheapness of prescribing antibiotics.

\section{Theme 2: Current clinical need in VAP diagnostics}

The interviews revealed that there is a clinical need for a diagnostic test that provides a more accurate and timely diagnosis of the causative pathogen (or lack of) and for disease monitoring, without the long delays associated with return of results (i.e. 24-72 hours for the BAL/PSB), and which is not dangerous to the patient (e.g. the transient reduction in oxygenation, associated with bronchoscopic procedures). When a VAP is present, a diagnostic test with these properties is expected to better facilitate the rationalisation and narrowing of antibiotic prescribing for patients with suspected VAP in the ICU, in comparison to current practice.

\section{Theme 3: The potential value and role of the OMA platform}

\section{The potential value of the OMA platform}

After discussion of current practice and clinical need, we then presented and discussed the OMA platform and its potential value and role in diagnosing a suspected VAP in the ICU.

It was indicated that the OMA platform's provision of the real-time gram information, by the bedside, could provide better rationalisation and narrowing of antibiotics-some antibiotics have more potency against Gram-positive than Gram-negative organisms, and vice versa. Stated differently, if the OMA platform can accurately differentiate between Gram-positive and negative organisms, then the initial empirical, broad spectrum antibiotics could be narrowed to the particular Gram classification.

Diagnostic tests targeting bacterial infections often give rise to a conceptual question over whether the bacteria detected represents infection or colonisation in the patient [43]; that is, whether the bacteria detected is causing disease or not, respectively-as the lung is non-sterile tissue and bacteria is expected. In addition to revealing the Gram-positive and/or Gram-negative bacteria in the patient lungs, the OMA platform can also identify activated neutrophils, which are associated with inflammation and infection. This information may help the OMA platform differentiate between colonisation and infection. 
The OMA platform provides explicit, real-time data through live video-feed to the patient's bedside. The bacteria and markers of inflammation can be visualised in vivo. It was suggested that this may be more powerful than surrogate markers in influencing decision making.

\section{The potential role of the OMA platform}

The interviews and the care pathway analysis indicated that the optimal role of the OMA platform would be as a replacement or new/add-on special investigation to diagnose VAP in the ICU. In ICUs that currently use BAL or PSB to diagnose VAP, the OMA platform would be a replacement. In ICUs that do not perform special investigations, the OMA platform would be a new test and an add-on to current practice. In both scenarios, the OMA platform would be used as a rule-in diagnostic, to facilitate rapid and Gram-targeted antibiotic treatment. There may be a secondary role for the OMA platform as a rule out diagnostic (less likely) and as a surveillance/monitoring device (less likely). These roles, and their advantages and disadvantages, are discussed below and are visually represented in Figure 2

\section{The OMA platform as a rule-in diagnostic}

The OMA platform, as a rule-in diagnostic, would be performed when there is a clinical suspicion of a VAP, when the patient is eligible for a bronchoscopic and transbronchial procedure (i.e. not physically or logistically contraindicated), and (ideally) prior to antibiotic treatment, to maximise the likelihood of visualising the bacteria and neutrophils, and performing a successful culture through use of the OMA platform's mini-lavage capabilities.

The real-time, Gram and neutrophil information from the OMA platform may allow the clinician to narrow their antibiotic prescription, from a broad spectrum, multi-therapy, empirical antibiotic to a Gram-tailored, (ideally) mono-therapy antibiotic. Assuming the OMA platform is sufficiently accurate, this has the potential to kill bacteria more effectively, reduce exposure of patients to unnecessary toxicity, reduce AMR, and reduce costs, since more effective treatment should reduce usage of ICU beds.

\section{The OMA platform as a rule-out diagnostic}

The OMA platform, as a rule-out diagnostic, would be performed when there is a clinical suspicion of a VAP, and when the patient is eligible for a bronchoscopic and transbronchial procedure.

The key advantage to ruling out VAP is that it potentially allows the clinical team to stop antibiotics, pushing them to explore alternative diagnoses for the patient. Also, there is a greater clinical need for 
a rule-out diagnostic, because the pathway to stop antibiotics is more complicated and less standardised across NHS hospitals, than is the pathway to start antibiotics.

The key disadvantage to this role-which is not unique to OMA platform, but extends to other rule out tests in NHS ICUs-is that ruling-out VAP does not always lead to the stopping or tailoring of antibiotics, because the patient might have an infection elsewhere in the body, including a section of the lung not sampled. As discussed Theme 1, there is a strong bias to continue antibiotics in ICU patients.

\section{The OMA platform as a surveillance and/or monitoring device}

The OMA platform as a surveillance device would be used as part of the routine investigations. As a monitoring device it would be used in patients that are being treated for a suspected VAP. In both scenarios, the patient must be eligible for a bronchoscopic and transbronchial procedure.

Using the OMA platform as a surveillance and/or monitoring device could have several advantages, but it was felt that the platform is likely too invasive and expensive to be used repeatedly in ICU patients. The platform, used in these roles, may be appropriate for other non-UK healthcare systems, which perform bronchoscopic procedures more routinely (e.g. parts of Europe, and large teaching hospitals in the US).

\section{Care pathway visualisation}

Figure 2: Visualisation of the pathway for diagnosing a suspected VAP in a UK NHS ICU. The coloured boxes in the pathway represent points at which the OMA platform could be used. The green box represents the optimal role for the device, based on the clinical interviews. Here, the OMA platform would be used as a rule in diagnostic. The orange boxes represent alternative possible roles for the OMA platform, as part of the routine investigations of all eligible ICU patients (top of the pathway) and as a monitoring test in patients with a suspected VAP (bottom of the pathway.

\section{Theme 4: Barriers to adoption}

\section{Training needs}

As with all new diagnostic tests, there will be training needs, but in this case the need is high because of the complexity and risks of the procedure (discussed in the next section). The training should cover the bronchoscopic procedure (additional trained clinicians may be required if the need for bronchoscopy increases a consequence of the platform introduction), the puncture of the interstitial lung tissue and alveoli (see next section), the administration of the smart probes, the interpretation of the smart probe data, and the collection of the mini-lavage. This would likely require senior respiratory physicians to perform the transbronchial puncture aspect of the procedure, but not all ICU consultants have a respiratory background. 
325

326

327

328

The transbronchial puncture aspect was highlighted as a potential risk to the patients, because of the risk of causing a pneumothorax. It was stated that the risk of causing a pneumothorax is probably quite low, but the risk level is dependent on the patient's characteristics. The following patients were identified as possessing a heightened risk of developing a pneumothorax: those patients with ARDS, COPD, sepsis, blood clotting abnormalities and patients requiring high ventilator pressure. It was highlighted that if the OMA platform were to cause a pneumothorax in the early stages of adoption in an ICU, it would likely not be used thereafter. The risk of transbronchial puncture is in addition to the transient interference of oxygenation caused by the bronchoscope, and would be increased further if the transbronchial puncture were performed in multiple sites of the lung. The platform's safety would have to be demonstrated formally and empirically. It is possible that the patients who would get the most out of this platform (e.g. those needing early tailoring of antibiotics) are those that are most at risk of developing complications from the procedure.

\section{Increased complexity and effort}

The OMA platform, as a bronchoscopic technique, adds an extra layer of complexity to diagnosis in comparison to non-bronchoscopic procedures (e.g. ETAs and N-BALS) and to a clinical diagnosis. The OMA platform requires a concerted effort to perform and interpret its results, and carries inherent risks to patients, therefore, there was some concern in the interviews that clinicians may not use this platform, unless strictly required to by guidelines.

\section{Cost}

The potential cost-saving element of the OMA platform, associated with more appropriate antibiotic prescribing (discussed in Theme 3), is likely to be attenuated by the labour-intensive and highly technical nature of the procedure.

\section{Theme 5: Evidence requirements to help facilitate successful adoption}

To feel confident using the OMA platform, the participants indicated that substantial, high quality evidence would be required on the diagnostic accuracy, clinical safety and cost-benefits.

It was indicated that the BAL may be a suitable reference standard for evaluating the diagnostic accuracy of the OMA platform. However, it was noted that clinicians who do not use BALs as their standard of care will be less persuaded to adopt the OMA platform if the study does not compare it with the test that they use (e.g. ETAs or N-BALS). Once the accuracy of the platform is adequately 
demonstrated, a clinical effectiveness/utility trial would be required, where patients would be randomised to the OMA platform or the comparator diagnostic.

Cost benefit/effectiveness will be required to help facilitate adoption of the OMA platform. The OMA platform and other bronchoscopic procedures cost more than the non-bronchoscopic procedures. Consequently, it will need to be demonstrated that the OMA platform provides value for money and is affordable, which could stem from better, more tailored patient care and reducing in ICU time.

\section{Discussion}

In this study we performed qualitative, semi-structured interviews with clinical experts. We used the information in these interviews to perform a care pathway analysis of current practice for diagnosing a suspected VAP in the UK NHS ICUs. We also explored the potential value and role of the OMA platform.

The care pathway analysis revealed that making a diagnosis of VAP is extremely difficult, primarily because the clinical signs associated with VAP overlap with several other diseases. To improve the accuracy and certainty of a VAP diagnosis, a special investigation may be performed (e.g. ETA, N-BAL, BAL, PSB), where, depending on the method, an upper or lower respiratory specimen is collected and sent for culture. These investigations varying in accuracy, invasiveness and cost. Time delays in receiving these results mean that the decision to treat (predominantly, with antibiotics), is based on clinical signs only. Consequently, antibiotics are typically empirical and broad spectrum.

The key driver for starting antibiotics is an overall, holistic deterioration in the clinical signs associated with the respiratory system. Antibiotics will be narrowed where possible, when microbiology becomes available. Antibiotics are usually started with a set duration, typically a 5- or 7-day course and this course is typically completed.

The key driving factor for stopping antibiotics is an overall, holistic improvement in the patient, based on the clinical signs associated with the respiratory system, including microbiological information. Deciding the exact point at which to stop antibiotics was reported to be extremely challenging, partly because there is no good rule-out test for pneumonia, and no standardised guidelines. It was also indicated that cognitive biases might affect the decision to stop antibiotics. Biases which have previously been demonstrated to be present in clinical decision making [44, 45], and antibiotic prescribing behaviour [46]. 
The interviews revealed that there is a clinical need for a diagnostic test that provides a more accurate and timely diagnosis of VAP, which is not dangerous to the patient, and which has properties that better facilitate the rationalisation and narrowing of antibiotic prescribing for patients.

Based on the care pathway analysis it was determined the OMA platform could potentially satisfy important aspects of the above clinical need. The OMA platform's provision of real-time gram information, by the bedside, could provide better rationalisation and narrowing of antibiotics, which may be more powerful than currently-used surrogate markers in influencing clinical decision making. Also, the platforms ability to identify activated neutrophils may also help differentiate between bacterial colonisation and infection. Although the focus of this study was VAP, these value propositions for the OMA platform may be equally applicable to all forms of bacterial pneumonia experienced in the ICU.

Diagnosing a VAP in the ICU is challenging. The initial suspicion of VAP is based on clinical signs associated with the respiratory system (including bloods and CXRs), which are not specific to VAP, and do not allow for the tailored antibiotic treatment. To improve the accuracy, or to reduce the uncertainty, requires an invasive diagnostic procedure, where an upper or lower respiratory specimen is collected and sent for microbiological testing. These procedures vary in safety, accuracy, and influence on clinical decision making.

There is a clinical need for a diagnostic test that provides a more accurate and timely diagnosis of the causative pathogen (or lack of) and for disease monitoring. When a VAP is present, a diagnostic test with these properties would better facilitate the rationalisation and narrowing of antibiotic prescribing in comparison to current practice. The care pathway analysis revealed that the OMA platform would address this aspect of the clinical need, but further evidence would be required on its accuracy, safety and cost-benefit.

Future recommendations

Further research into the cognitive biases involved in antibiotic decision making in the ICU would be informative for clinicians and developers. 
413 Ethical approval was obtained from the HRA to carry out interviews on NHS staff (IRAS number: 414 242651).

415 Consent for publication

416 Not applicable.

418 Key quotes from the interviews are provided in the Online Appendices. No additional data are 419 available.

\section{Competing interests}

$421 \mathrm{KD}, \mathrm{AB}$, and JM have competing interests. They are involved in the development of the technology under review. Their contributions were restricted to fact-checking of the technology description, and they also helped us with the conceptualisation of the initial study design. This study was performed, analysed and reported by the NIHR Newcastle In Vitro Diagnostics Co-operative (Newcastle MIC), which is an independent group, funded by NIHR, to generate high-quality evidence on diagnostic devices. The Newcastle MIC had full control of the content in the manuscript.

\section{$427 \quad$ Funding}

428 This study was funded by a CARB-X grant (4500002330). The NIHR funds the NIHR Newcastle In Vitro 429 Diagnostics Co-operative.

\section{$430 \quad$ Authors' contributions}

431 All authors made substantial contributions to the design of the study. WSJ, JS, AJA and SG performed 432 and analysed the clinical interviews. WSJ drafted the manuscript for this study. All authors critically 433 reviewed iterative drafts of revisions of the manuscript, and approved the final version.

\section{Acknowledgements}

435 We would like to thank the clinical experts that contributed their time in participating in the 436 interviews. These Individuals will remain anonymous. 
438 1. The National Institute for Health and Care Excellence (NICE), Pneumonia in adults: diagnosis and management. 2019.

2. Anand, N. and M.H. Kollef, The alphabet soup of pneumonia: CAP, HAP, HCAP, NHAP, and VAP. Semin Respir Crit Care Med, 2009. 30(1): p. 3-9.

3. Koulenti, D., E. Tsigou, and J. Rello, Nosocomial pneumonia in 27 ICUs in Europe: perspectives from the EU-VAP/CAP study. Eur I Clin Microbiol Infect Dis, 2017. 36(11): p. 1999-2006.

4. Niederman, M.S., Hospital-acquired pneumonia, health care-associated pneumonia, ventilatorassociated pneumonia, and ventilator-associated tracheobronchitis: definitions and challenges in trial design. Clin Infect Dis, 2010. 51 Suppl 1: p. S12-7.

5. Ferrer, M. and A. Torres, Epidemiology of ICU-acquired pneumonia. Curr Opin Crit Care, 2018. 24(5): p. 325-331.

6. Spencer, R.C., Epidemiology of infection in ICUs. Intensive Care Med, 1994. 20 Suppl 4: p. S2-6.

7. Vallés, J., et al., Excess ICU mortality attributable to ventilator-associated pneumonia: the role of early vs late onset. Intensive Care Med, 2007. 33(8): p. 1363-8.

8. Heyland, D.K., et al., The attributable morbidity and mortality of ventilator-associated pneumonia in the critically ill patient. The Canadian Critical Trials Group. Am J Respir Crit Care Med, 1999. 159(4 Pt 1): p. 1249-56.

9. Safdar, N., et al., Clinical and economic consequences of ventilator-associated pneumonia: a systematic review. Crit Care Med, 2005. 33(10): p. 2184-93.

10. Kollef, M.H., C.W. Hamilton, and F.R. Ernst, Economic impact of ventilator-associated pneumonia in a large matched cohort. Infect Control Hosp Epidemiol, 2012. 33(3): p. 250-6.

11. Rello, J., et al., Epidemiology and outcomes of ventilator-associated pneumonia in a large US database. Chest, 2002. 122(6): p. 2115-21.

12. Balogun, B., Coronavirus: Ventilator availability in the UK, H.o.C. Library, Editor. 2020.

13. Mahase, E., Covid-19: most patients require mechanical ventilation in first 24 hours of critical care. BMJ, 2020. 368: p. m1201.

14. Akram, A.R., et al., In situ identification of Gram-negative bacteria in human lungs using a topical fluorescent peptide targeting lipid A. Sci Transl Med, 2018. 10(464).

15. Chan, Y.R. and A. Morris, Molecular diagnostic methods in pneumonia. Curr Opin Infect Dis, 2007. 20(2): p. 157-64.

16. Drabińska, N., et al., From fast identification to resistance testing: Volatile compound profiling as a novel diagnostic tool for detection of antibiotic susceptibility. TrAC Trends in Analytical Chemistry, 2019. 115: p. 1-12.

17. Hellyer, T. and J. Simpson, Biomarker-based exclusion of ventilator-associated pneumonia: a multicentre validation study. Critical Care, 2014. 18(Suppl 1): p. P303-P303.

18. Hellyer, T.P., et al., Diagnostic accuracy of pulmonary host inflammatory mediators in the exclusion of ventilator-acquired pneumonia. Thorax, 2015. 70(1): p. 41.

19. Jung, J.H. and J.E. Lee, Real-time bacterial microcolony counting using on-chip microscopy. Scientific reports, 2016. 6: p. 21473-21473.

20. Mills, B., M. Bradley, and K. Dhaliwal, Optical imaging of bacterial infections. Clinical and translational imaging, 2016. 4: p. 163-174.

21. Morris, A.C., Management of pneumonia in intensive care. J Emerg Crit Care Med, 2018.

22. Slupsky, C.M., Nuclear magnetic resonance-based analysis of urine for the rapid etiological diagnosis of pneumonia. Expert Opinion on Medical Diagnostics, 2011. 5(1): p. 63-73.

23. The Proteus Interdisciplinary Research Collaboration is funded by the Engineering and Physical Sciences Research Council (EPSRC). 2017; Available from: https://proteus.ac.uk/about/theconsortium/. 
24. Korevaar, D.A., et al., Targeted test evaluation: a framework for designing diagnostic accuracy studies with clear study hypotheses. Diagnostic and Prognostic Research, 2019. 3(1): p. 22.

25. Charman, S., et al., Opportunities and challenges of a novel cardiac output response to stress (CORS) test to enhance diagnosis of heart failure in primary care: qualitative study. BMJ Open, 2019. 9(4): p. e028122.

26. De Bleser, L., et al., Defining pathways. J Nurs Manag, 2006. 14(7): p. 553-63.

27. Graziadio, S., et al., How to Ease the Pain of Taking a Diagnostic Point of Care Test to the Market: A Framework for Evidence Development. Micromachines (Basel), 2020. 11(3).

28. Kinsman, L., et al., What is a clinical pathway? Development of a definition to inform the debate. BMC Med, 2010. 8: p. 31.

29. Panella, M. and K. Vanhaecht, Is there still need for confusion about pathways? International Journal of Care Pathways, 2010. 14(1): p. 1-3.

30. Saunders, B., et al., Saturation in qualitative research: exploring its conceptualization and operationalization. Qual Quant, 2018. 52(4): p. 1893-1907.

31. Braun, V. and V. Clarke, Using thematic analysis in psychology. Qualitative Research in Psychology, 2006. 3(2): p. 77-101.

32. Castleberry, A. and A. Nolen, Thematic analysis of qualitative research data: Is it as easy as it sounds? Curr Pharm Teach Learn, 2018. 10(6): p. 807-815.

33. Maguire, M. and B. Delahunt. Doing a thematic analysis: A practical, step-by-step guide for learning and teaching scholars. 2017.

34. Nowell, L.S., et al., Thematic Analysis:Striving to Meet the Trustworthiness Criteria. International Journal of Qualitative Methods, 2017. 16(1): p. 1609406917733847.

35. Gale, N.K., et al., Using the framework method for the analysis of qualitative data in multidisciplinary health research. BMC Medical Research Methodology, 2013. 13(1): p. 117.

36. Pugin, J., et al., Diagnosis of ventilator-associated pneumonia by bacteriologic analysis of bronchoscopic and nonbronchoscopic "blind" bronchoalveolar lavage fluid. Am Rev Respir Dis, 1991. 143(5 Pt 1): p. 1121-9.

37. Kalil, A.C., et al., Management of Adults With Hospital-acquired and Ventilator-associated Pneumonia: 2016 Clinical Practice Guidelines by the Infectious Diseases Society of America and the American Thoracic Society. Clinical Infectious Diseases, 2016. 63(5): p. e61-e111.

38. Shan, J., H.L. Chen, and J.H. Zhu, Diagnostic accuracy of clinical pulmonary infection score for ventilator-associated pneumonia: a meta-analysis. Respir Care, 2011. 56(8): p. 1087-94.

39. Walsh, T.S., A.C. Morris, and A.J. Simpson, V. Ventilator associated pneumonia: can we ensure that a quality indicator does not become a game of chance? BJA: British Journal of Anaesthesia, 2013. 111(3): p. 333-337.

40. Craven, T.H., et al., Ventilator-associated pneumonia surveillance using two methods. Journal of Hospital Infection, 2020. 104(4): p. 522-528.

41. Craven, T.H., et al., Lack of concordance between ECDC and CDC systems for surveillance of ventilator associated pneumonia. Intensive Care Medicine, 2018. 44(2): p. 265-266.

42. Arulkumaran, N., et al., Antimicrobial-associated harm in critical care: a narrative review. Intensive Care Med, 2020. 46(2): p. 225-235.

43. Dani, A., Colonization and infection. Central European journal of urology, 2014. 67(1): p. 86-87.

44. O'Sullivan, E.D. and S.J. Schofield, Cognitive bias in clinical medicine. J R Coll Physicians Edinb, 2018. 48(3): p. 225-232.

45. Saposnik, G., et al., Cognitive biases associated with medical decisions: a systematic review. BMC medical informatics and decision making, 2016. 16(1): p. 138-138.

46. Warreman, E.B., et al., Determinants of in-hospital antibiotic prescription behaviour: a systematic review and formation of a comprehensive framework. Clin Microbiol Infect, 2019. 25(5): p. 538-545. 


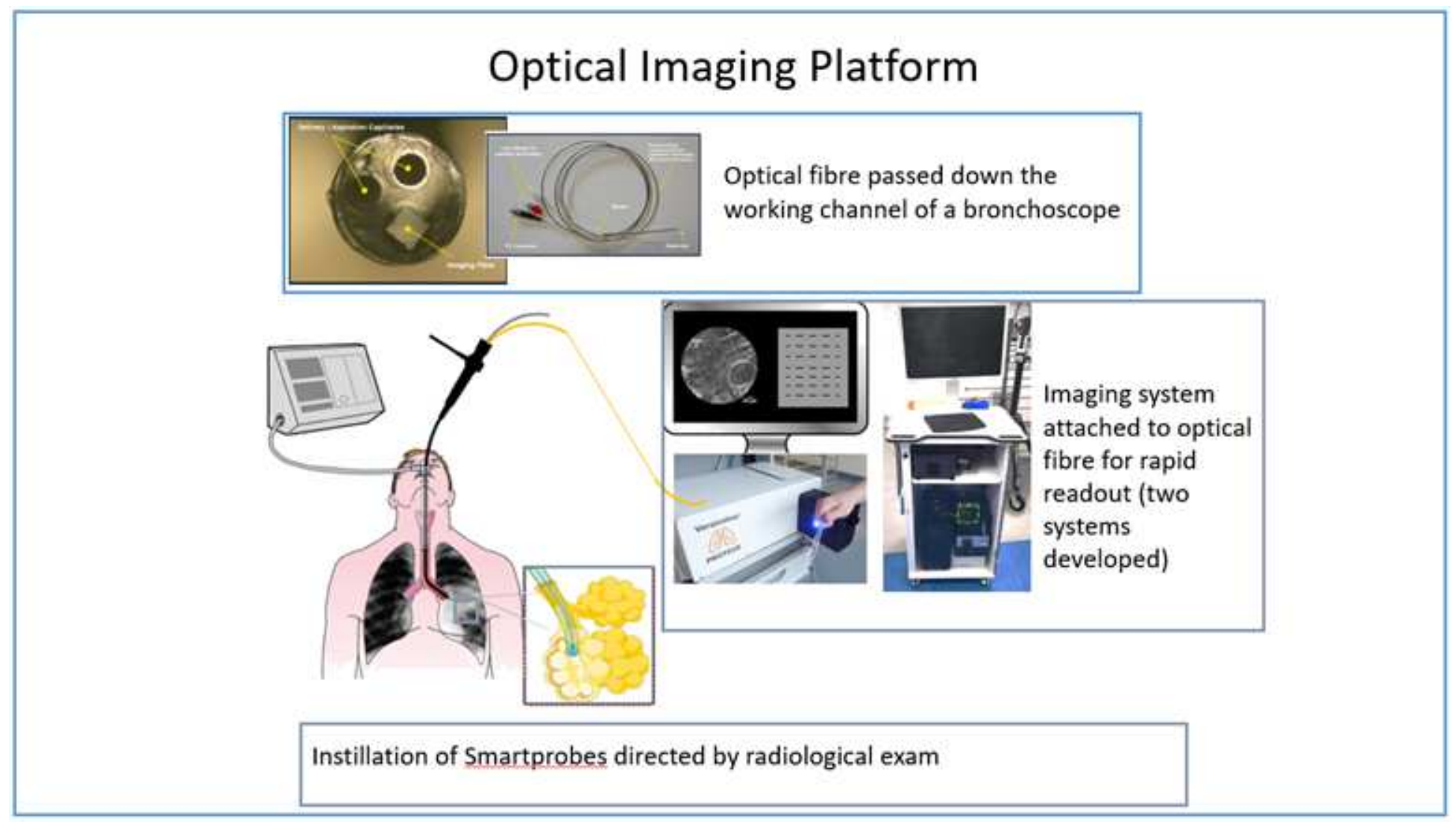

\section{Figure 1}

Image showing a bronchoscopy procedure with the imaging fibre and capillary bundle being passed down the working channel of a bronchoscope into the alveolar regions. SmartProbes are delivered via the capillaries and images are sent via the imaging fibre to the imaging system at the patient's bedside. 


\begin{tabular}{|l|}
\hline Key: \\
$\mathrm{ABX}=$ antibiotics \\
$\mathrm{BAL}=$ bronchoalveolar lavage \\
$\mathrm{CT}=$ computerised tomography \\
$\mathrm{CXR}=$ chest $x$-ray \\
$\mathrm{ET}=$ endotracheal tube \\
$\mathrm{ETA}=$ endotracheal aspirate \\
$\mathrm{ICU}=$ intensive care unit \\
$\mathrm{N}-\mathrm{BAL}=$ non-bronchoscopic $\mathrm{BAL}$ \\
$\mathrm{PSB}=$ protected specimen brush \\
WCC = white cell count
\end{tabular}

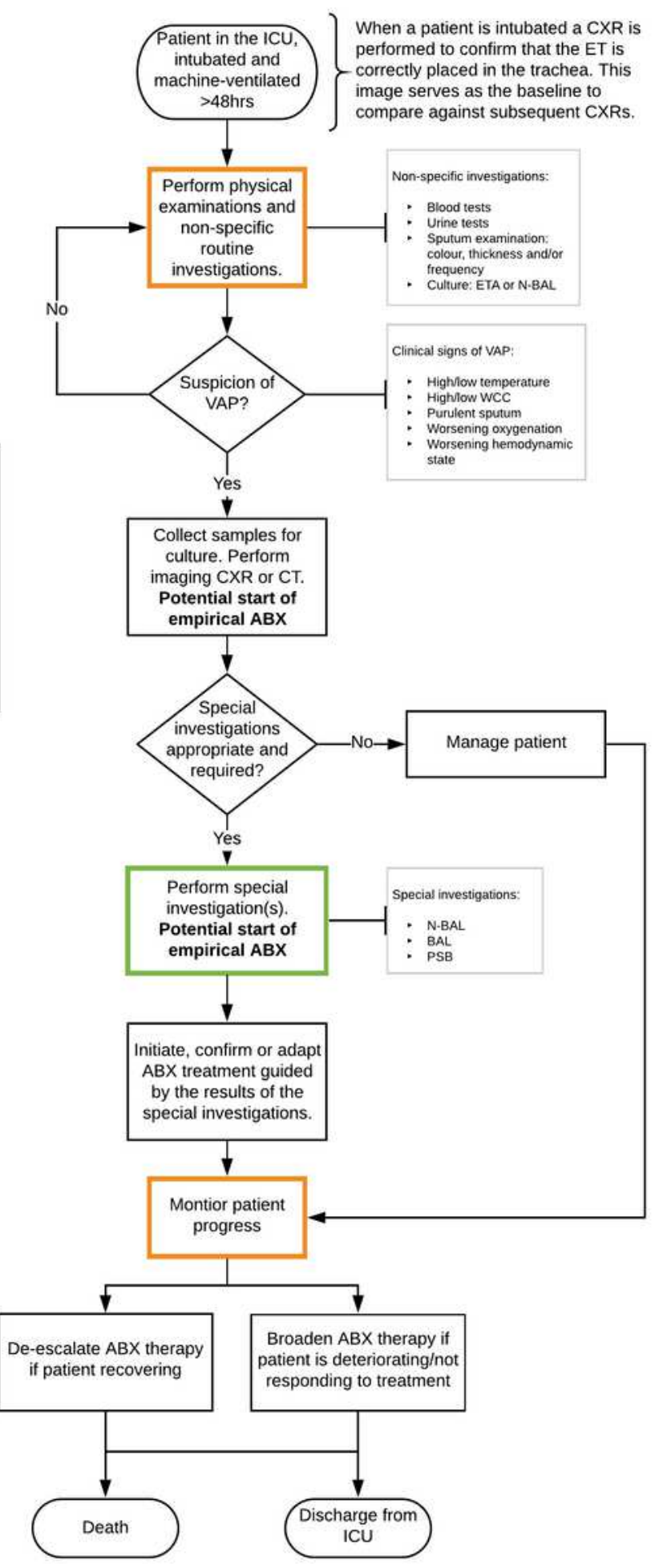

\section{Figure 2}

Visualisation of the pathway for diagnosing a suspected VAP in a UK NHS ICU. The coloured boxes in the pathway represent points at which the OMA platform could be used. The green box represents the optimal role for the device, based on the clinical interviews. Here, the OMA platform would be used as a rule in diagnostic. The orange boxes represent alternative possible roles for the OMA platform, as part of 
the routine investigations of all eligible ICU patients (top of the pathway) and as a monitoring test in patients with a suspected VAP (bottom of the pathway.

\section{Supplementary Files}

This is a list of supplementary files associated with this preprint. Click to download.

- OMAQualVAPManuscriptAppendicesv02.pdf 